\title{
Skin Cancer Detection Using Temperature Variation Analysis
}

\author{
Ahmed M. Nasr Moustafa ${ }^{1}$, Hamed Hamid Muhammed ${ }^{1}$, Moustapha Hassan ${ }^{2}$ \\ ${ }^{1}$ Division of Informatics, Logistics and Management, School of Technology and Health STH, \\ Royal Institute of Technology KTH, Stockholm, Sweden \\ ${ }^{2}$ Clinical Research Center Novum, Karolinska University Hospital Huddinge, Stockholm, Sweden \\ Email: amnmo@kth.se, Hamed.Muhammed@sth.kth.se, Moustapha.Hassan@ki.se
}

Received September 2012

\begin{abstract}
In the medical field, new technologies are incorporated for the sole purpose of enhancing the quality of life for the patients and even for the normal healthy people. Infrared technology is one of the technologies that have some applications in both the medical and biological fields. In this work, the thermal infrared (IR) measurement is used to investigate the potential of skin cancer detection. IR enjoys non-invasive and non-contact advantages as well as favorable cost, apparently. It is also very well developed regarding the technological and methodological aspects. IR per se is an electrometric radiation that all objects emit when their temperature is above the absolute zero. And the human body is not different in this regard. The IR range extends, ideally, to cover wavelengths from 800 nanometer to few hundred micrometer. Cancer, in modern life, has grown tangibly due to many factors, such as life expectancies increase, personal habits and ultraviolet radiation exposures among others. Moreover, the significant enhancement of technologies has helped identifying more types of cancers than before. The sole purpose of this work is to investigate further IR technology methods and applications not yet matured in skin cancer detection to enhance the detection ability with higher safety level.
\end{abstract}

Keywords: Infrared; Skin Cancer; Melanoma; Thermal; Detection

\section{Introduction}

Every object above absolute zero temperature $(0 \mathrm{~K})$ emits infrared (IR) electromagnetic radiation. In human skin, it is mostly the blood circulation and cells' metabolic activity that produce heat [6]. The emissivity of the human skin can be approximated as a true black body with unity value $[2,6]$. The natural emission of thermal infrared (IR) from the human body adds an important advantage to any approach that is based on measuring and analyzing this natural thermal emission. This makes such a technique clearly superior to any other invasive approach.

What makes things even more feasible are probably the cancer properties themselves. The regular definition of cancer is likely to contain an indication to abnormal growth of cells due to changes in gene expression which causes loss of control exerted normally on human cells. This leads to a profound effect on cell characteristics such as proliferation rate, size, shape, etc. Furthermore, cancer is regularly accompanied with angiogenesis $[3,4]$; i.e. new formation of blood vessels to supply enough nutrition and oxygen to the cancerous area. The aforementioned in short suggests that cancerous cells can stand out when thermal infrared (IR) emission is involved. In addition, IR radiation from human skin seems to be accompanied also with functional properties [2]. This is considered as an advantage that is always welcomed.

- The skin is known to be a vital organ for the human body. It is also the largest organ of the body, which makes out one-third of a normal person's total body weight [1]. It is, naturally, responsible for many biological functions as well as its inherent protection functions such as sensory functions, and metabolism of nutrition such as vitamin D3 $[1,3]$. The skin is mainly divided into three layers: the epidermis, the dermis and the hypodermis. The outermost layer is the epidermis. The innermost layer is the subcutaneous layer, also called hypodermis. The dermis is the middle layer that is sandwiched between the epidermis and the hypodermis.

- Millions of skin cancer cases are recorded every year around the world $[1,5,8]$. It is probably the most common cancer that can be developed in a human being. Skin cancer per se is a type of epithelial tissue cancer. It can be both benign and malignant. Since 
skin has easy access to both the nearby lymphatic system as well as the nearby blood stream, malignant types can produce metastasis that are easily transported and spread to other organs and tissues in the body and become extremely lethal. Actually it is these metastases that can cause most deaths from skin cancer.

- Melanoma is the most aggressive type of skin cancer that is considered to be highly malignant and fatal. Melanoma usually originates in many cases in moles, which are intensive clusters of melanocytes [1]. Two other well-known forms of skin cancer are: basal cell carcinoma (BCC) and squamous cell carcinoma (SCC). Both of them are, usually, less harmful than melanoma. BCC is the most common form of skin cancer [1,3]. There is also a high risk that these types extend and produce harmful effect on their neighborhood. In rare cases they can become malignant and metastastic [1]. This is a risk that is normally taken into account since they become aggressive and life threatening.

\section{Methods}

In this work, anon contact IR temperature sensor is used to test the ability of the detection of the presence of skin cancer. Human skin is often referred to as a black body as mentioned above. The main range of measurement for the current study has IR-radiation wavelengths of 8 - 14 $\mu \mathrm{m}$. The used technique is a noncontact localized detection and is thought to be advantageous mainly because of black body radiation has nearly $40 \%$ of its emitted
IR-radiation power within this specific spectral region at the corresponding temperature of the skin [10]; i.e. the largest percentage of the radiated power. This can makes it easier for the detection of anomalies in IR radiation from the skin in the presence of skin cancer. Moreover, at the used detection wavelengths, IR radiation suffers nearly no absorption from air gas molecules [11], which constitutes another advantage.

Two laboratory animals were used for the actual measurements in this work. Each lab animal (mouse) was injected with cancerous cells under the skin at an arbitrary spot and allowed to develop skin cancer. The area around the injection spot was shaved as well as another healthy area to be used as a normal reference was also shaved. After several days, the skin cancer was developed and became visually apparent. Thereafter, the measurement process was conducted. Figure 1 portrays a simplified schematic of the experimental setup. The two animals used were well restrained and motionless during the procedure by proper means used in the laboratory.

Two non-contact thermal IR sensors from Optris GMBH were used concurrently. These sensors were identical; i.e. from the same manufacturer and of the same type. Figure 2 shows an image for the sensor type that was used. The sensors had the ability to record 1000 measurements per second and had a resolution of $0.1^{\circ} \mathrm{C}$. One of the sensors was used to measure the temperature variation of a healthy area of the skin and the other one was used to measure the temperature variation due to the heat emission from the cancerous area. The measure-

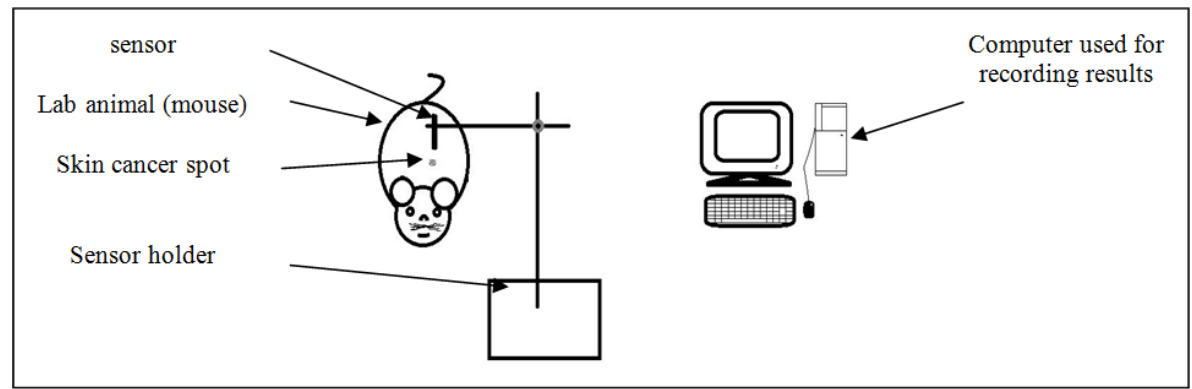

Figure 1. A simplified schematic of the measurement setup of the thermal IR emission from the lab animal (mouse) and direct storage to a computer.

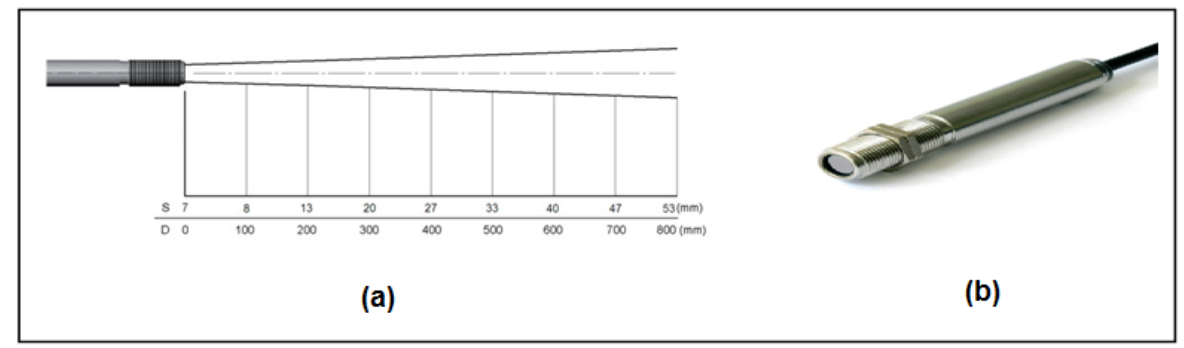

Figure 2. (a) Detection field of view size vs. distance from the IR sensor (D is the diameter of the field-of-viewspot, $S$ is the distance from the top of the IR sensor); (b) An image of the sensing head of the used IR sensor. 
ments were performed simultaneously to obtain a form of a differential overview that coincided well in time. The measurements were conducted for a sufficient period of time and stored for later analysis and investigation.

It is worth mentioning that each sensor was placed directly above the measured spot at a close distance of 1 $\mathrm{cm}$ to form a direct well-localized measurement and to maximize a solid IR detection. The sensors were connected simultaneously to a computer and had the capability of storing two long lists of temperature measurements vs. the time.

\section{Results and Discussion}

The obtained experimental results were in the form of tabulated temperature measurements for the healthy skin area and the cancerous area in the skin. Both sets of measurements were simultaneously conducted to achieve synchronization. These results were stored on the computer internal storage.

The first step of analyzing the acquired data was to compute a number of statistical parameters for the healthy and the cancerous skin areas for each lab animal. Thereafter, the next step was to qualitatively compare these parameters in an efficient and reliable way.

Statistical tests, such as the student-test as well as the ANOVA test were also used to be able to see if it was possible to differentiate between the IR emission measurements acquired on a healthy spot and the corresponding measurements that were acquired on a skin cancer spot.

Table 1 displays the results for a sample dataset of ten measurements for the cancerous spot in one animal. The columns of the table in this figure contain the calculated values for the mean, median, standard deviation, maximum, minimum as well as the difference between the maximum and the minimum values of the ten-measurements sample dataset corresponding to a sufficient period of time.

Table 2 shows the corresponding calculated values for ten concurrent measurements for a healthy spot that is used as a comparison reference in the same lab animal.

Preliminary results, of a quantitative comparison between the values of the parameters presented in these two tables, indicate that there exists a tangible difference between the mean and the median temperature values that can differentiate between the cancerous region and the corresponding healthy region of the skin in the same animal. All other calculated parameters, such as the standard deviation, maximum, minimum, etc., had no apparent advantage in this context.

The two statistical analysis tasks (the pair-wise student t-test and the pair-wise ANOVA test) were performed, where 1000 acquired measurements were used at each time, not only the small subset of ten measurements that were used to calculate the values presented in the tables.

The student t-test was conducted pair-wise between each dataset of 1000 measurements of the cancerous region in one of the two used lab animals and the corresponding dataset of 1000 measurements from the healthy skin spot in the same lab animal.

The t-test analysis results showed a significant difference between the datasets of 1000 measurements obtained from each pair of a healthy skin region and the corresponding cancerous one in each lab animal. As a result, p-value levels around 0.05 was obtained in both lab animals (actually even better, i.e. lower, p-values

Table 1. Calculations performed on ten measurements (columns) each for a similar period of time in the cancerous spot (all number are in ${ }^{\circ} \mathrm{C}$ ).

\begin{tabular}{|c|c|c|c|c|c|c|c|c|c|c|}
\hline Mean & 31.94 & 31.76 & 31.96 & 31.72 & 31.81 & 31.89 & 31.97 & 32.03 & 32.04 & 32.05 \\
\hline Median & 31.9 & 31.8 & 32 & 31.7 & 31.8 & 31.9 & 32 & 32 & 32 & 32.1 \\
\hline StdDev & 0.23 & 0.26 & 0.21 & 0.21 & 0.20 & 0.26 & 0.20 & 0.23 & 0.26 & 0.21 \\
\hline Max & 32.5 & 32.5 & 32.6 & 32.2 & 32.3 & 32.6 & 32.5 & 32.7 & 32.7 & 32.6 \\
\hline Min & 31.3 & 31.2 & 31.2 & 31.1 & 31.2 & 31.2 & 31.3 & 31.1 & 31.2 & 31.5 \\
\hline Max-Min & 1.2 & 1.3 & 1.4 & 1.1 & 1.1 & 1.4 & 1.2 & 1.6 & 1.5 & 1.1 \\
\hline
\end{tabular}

Table 2. Calculations performed on the corresponding ten measurements (columns) each for a similar period of time in the normal spot (all number are in ${ }^{\circ} \mathrm{C}$ ).

\begin{tabular}{cccccccccccc}
\hline mean & 31.44 & 31.45 & 31.32 & 31.31 & 31.38 & 31.30 & 31.19 & 31.24 & 31.27 & 31.24 \\
median & 31.4 & 31.4 & 31.3 & 31.3 & 31.4 & 31.3 & 31.2 & 31.3 & 31.3 & 31.2 \\
StdDev & 0.23 & 0.21 & 0.23 & 0.25 & 0.20 & 0.24 & 0.24 & 0.21 & 0.21 & 0.23 \\
Max & 32 & 32.4 & 31.9 & 32.1 & 32 & 31.9 & 31.9 & 31.9 & 31.9 & 31.9 \\
Min & 30.9 & 30.9 & 30.7 & 30.5 & 30.9 & 30.4 & 30.5 & 30.6 & 30.6 & 30.5 \\
Max-Min & 1.1 & 1.5 & 1.2 & 1.6 & 1.1 & 1.5 & 1.4 & 1.3 & 1.3 & 1.4 \\
\hline
\end{tabular}


were obtained).

These results would lead to a strong indication that thermal IR sensors (operating within the wavelength range 8 - $14 \mu \mathrm{m}$ ) can be used to locally detect and identify an area of cancer on the skin (in comparison to a neighboring normal healthy skin spot). Both animals showed this trend in the conducted student t-test.

The other statistical test, that was used to investigate the significance of using the acquired IR emission measurements, was ANOVA. Pair-wise ANOVA was conducted in a similar fashion for datasets of 1000 measurements from both animals. This test served to strengthen the significance of our experimental approach; that the difference in temperature measured using thermal IR radiation within the wavelength range $8-14 \mu \mathrm{m}$ was not due to chance, but rather to real variation between healthy and cancerous regions of the lab animals under investigation.

\section{Conclusion}

The results and the conducted analysis performed in this work suggest that a significant difference between the thermal IR radiation from healthy and cancerous skin regions shows potential for discriminating cancerous spots on the skin against healthy ones. Therefore it can be concluded that there is strong evidence that temperature distribution measured can be a highly sufficient indication for cancerous regions in the skin of the lab animals. This however might need further work to develop into an established technique which will occur in the future.

\section{REFERENCES}

[1] P.-L. So, “Skin Cancer,” Infobase Publishing, New York, 2008.

[2] M. Anbar, "Clinical Thermal Imaging Today, Shifing from Phenomenological Thermography to Pathophysiologically Based Thermal Imaging," IEEE Engineering in Medicine and Biologys, 1998, pp. 25-38.

[3] K. Nouri, “Skin Cancer,” McGraw Hill, New York, 2008.

[4] D. Marmé, “Tumor Angiogenesis,” 2008.

[5] R. W. Ruddon, “Cancer Biology,” Oxford University Press, Inc., Oxford, 2007.

[6] R. F. Schmidt, G. Thews, et al., "Human Physiology," Springer-Verlag, Berlin, 1989.

[7] A. Sunds, "Nya Behandlingar Bromsas av Regler,” Biotech Sweden, 7 November 2012, pp. 14-15.

[8] The Swedish Cancer Society. www.cancerfonden.se

[9] B. B. Lahiri, S. Bagavathiappan, T. Jayakumar and J. Philip, "Medical Applications of Infrared Thermography: A Review," Infrared Physics \& Technology, Vol. 55, 2012, pp. 221-235. http://dx.doi.org/10.1016/j.infrared.2012.03.007

[10] N. Diakides and J. Bronzino, "Medical Infrared Imaging," CRC Press, New York, 2008.

[11] M. Vollmer and K.-P. Möllmann, "Infrared Thermal Imaging: Fundamentals, Research and Applications,” Wiley-VCH Verlag, GmbH \& Co. KGaA, Weinheim, 2010. 